JPPT I Clinical Investigation

\title{
Uncomplicated Urinary Tract Infection in Ambulatory Primary Care Pediatrics: Are We Using Antibiotics Appropriately?
}

Ban AL-Sayyed, MD; Jeremy Le, MD; Mohammad Mousbah Al-Tabbaa, MD; Brian Barnacle, BS; Jinma Ren, PhD; Richard Tapping, PhD; and Meenakshy Aiyer, MD

OBJECTIVES Antimicrobial stewardship programs target antimicrobial use within the inpatient care setting. However, most antimicrobials are prescribed at ambulatory sites. We aim to determine the appropriateness of the diagnosis and treatment of uncomplicated urinary tract infection (UTI) in children within the outpatient setting at our institution, and to evaluate the cost of antibiotic treatment in our patient cohort.

METHODS This retrospective study was conducted by reviewing electronic records of patients aged 2 to 18 years diagnosed with uncomplicated UTI and treated with antibiotics in the outpatient setting from January 1, 2016, to April 30, 2016. Appropriate diagnosis was defined as confirmed UTI that included: pyuria $>5$ white blood cells per high-power field or positive for leukocyte esterase), a positive urine culture ( $\geq 50,000$ colony units $/ \mathrm{mL}$ of a single uropathogen for a catheterized sample or $\geq 100,000$ colony units $/ \mathrm{mL}$ for a clean catch urine sample), and lower urinary tract symptoms. Treatment was considered appropriate if the patient was prescribed first-line antibiotic for the susceptible isolate (trimethoprim sulfamethoxazole, amoxicillinclavulanate, nitrofurantoin, and cephalexin), and if the appropriate dose was used.

RESULTS We included 178 patients receiving a diagnosis of uncomplicated UTI and treated with antibiotics. Of these, $70 \%$ received an inappropriate diagnosis $(n=125) .58 \%(n=72)$ of improperly diagnosed patients had polymicrobial growth in their urine cultures. Antibiotics prescribed mostly in this group were trimethoprim-sulfamethoxazole $(53 \%, n=66)$ and cephalexin $(22 \%, n=27)$. Only $30 \%$ of all included patients received an appropriate diagnosis $(n=53)$. Of all appropriate diagnosis patients $(n=53), 26 \%$ were treated inappropriately $(n=14)$ with either wide-spectrum antibiotics $(n=8)$ or with low calculated dose $(n=6)$. The estimated cost of antibiotic treatment for the inappropriate diagnosis group $(n=125)$ was $\$ 10,755.87$.

CONCLUSION Antimicrobial stewardship programs should target the pediatric outpatient setting and antibiograms should be developed. Education of providers about the appropriate diagnosis and treatment of uncomplicated UTI in children is essential for reducing the cost of inappropriate therapy.

ABBREVIATIONS ASPS, antimicrobial stewardship programs; MIC, minimal inhibitory concentration; TMPSMX, trimethoprim-sulfamethoxazole; UTI, urinary tract infection

KEYWORDS ambulatory primary care pediatrics; inappropriate diagnosis of UTI; inappropriate use of antibiotics; outpatient antibiotic stewardship; uncomplicated urinary tract infection in children

J Pediatr Pharmacol Ther 2019;24(1):39-44

DOI: 10.5863/1551-6776-24.1.39

\section{Introduction}

Urinary tract infections (UTIs) are estimated to affect around $2.8 \%$ of children every year in the United States, which accounts for almost 1.1 million outpatient visits annually. The presumptive diagnosis of UTI is based on clinical symptoms in pediatric patients, which leads providers to prescribe antibiotics while awaiting confirmatory urine cultures. ${ }^{2,3}$ Ambulatory care providers tend to prescribe broad-spectrum antibiotics for UTI, with a substantial increase in prescription of third-generation cephalosporins on a national level. ${ }^{4}$ According to the Centers for Disease Control and Prevention, 30\% of prescribed antibiotics in the outpatient setting are unnecessary, and total inappropriate antibiotic use approaches $50 \%$ of all outpatient antibiotic use. ${ }^{5}$ Misuse of antibiotics in general can be secondary to inappropriate indication, choice, dose, and length of therapy. ${ }^{6-8}$ In 2009, around $\$ 10.7$ billion was spent on antibiotic therapy in the United States, including $\$ 6.5$ billion in the outpatient setting. ${ }^{9}$

Antimicrobial stewardship programs (ASPs) have proved to reduce antimicrobial misuse and bacterial resistance, improve patient safety and outcomes, and decrease health care-associated infections. ${ }^{10}$ Most ASPs target antimicrobial use within the inpatient care setting. ${ }^{11-16}$ However, most antimicrobials are prescribed 
Figure 1. Flowchart demonstrating the process of selection of outpatient case.

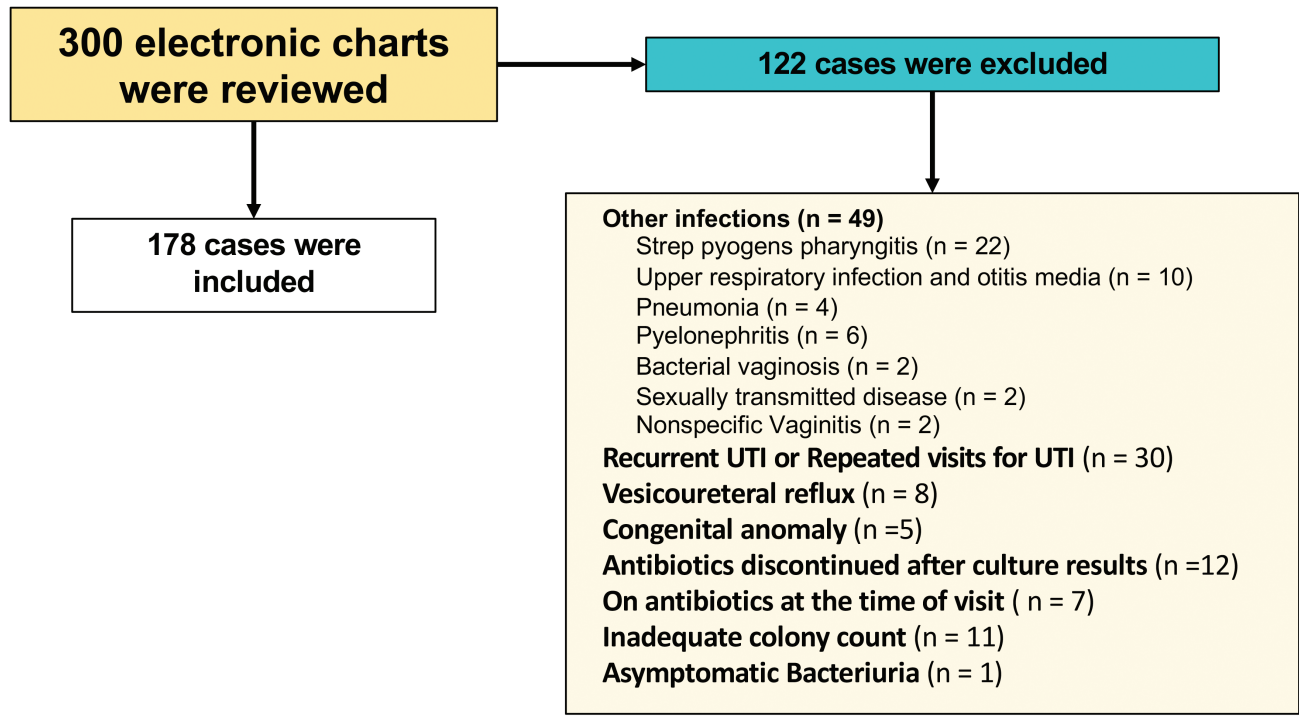

UTI, urinary tract infection.

at ambulatory care sites, making these sites another essential target for ASPs. ${ }^{17,18}$ The aim of this study is to assess the appropriateness of diagnosis and antibiotic treatment in uncomplicated UTI within ambulatory pediatric clinics, prompt care sites, and pediatric emergency room in our institution, and therefore emphasize the need for developing ASPs in our outpatient sites.

\section{Materials and Methods}

Study Design and Population. This cohort study was a retrospective analysis of pediatric patients receiving a diagnosis of uncomplicated UTIs in the Children's Hospital of Illinois primary care pediatrics, OSF prompt care and primary care clinics, and pediatric emergency room from January 1, 2016, to April 30, 2016. Patients aged 2 to 18 years, receiving a diagnosis of uncomplicated UTI and treated with antibiotics, were identified using the Epic electronic computerized database. An appropriate diagnosis of uncomplicated UTI was defined as pyuria (>5 white blood cells per high-power field or dipstick positive for leukocyte esterase), evidence of significant bacteriuria in the form of positive urine culture $(\geq 50,000$ colony-forming units $/ \mathrm{mL}$ of a single uropathogen for a catheterized sample or $\geq 100,000$ colony-forming units/ $\mathrm{mL}$ of a single uropathogen for a clean catch urine sample), and lower urinary tract symptoms. ${ }^{19,20}$ Frequency, urgency, dysuria, malodor of urine, secondary enuresis, and suprapubic pain were considered positive symptoms for lower UTI. Bacteriuria was suggested by positive nitrites on dipstick analysis, or any bacteria per high-power field on standard microscopic analysis (centrifuged and unstained), or any bacteria per 10 oil immersion fields on a Gram-stained smear on enhanced urinalysis. ${ }^{21}$ Patients who were included were categorized into inappropriate diagnosis and appropriate diagnosis groups according to finalized urine culture results after 48 hours from the initial visit. Inappropriate diagnosis patients were considered inappropriately treated. Appropriate diagnosis patients were divided into appropriately treated and inappropriately treated groups according to antibiotic choice and dose, history of allergy, de-escalation after isolate identification, and tolerance of the prescribed antibiotic.

Inclusion and Exclusion Criteria. Cases of uncomplicated UTI were defined as: any visit assigned an International Classification of Diseases, 9th revision and 10th revision, Clinical Modification diagnosis code of UTI, acute cystitis, dysuria, frequency of urination, urine incontinence-enuresis, and urine malodor. We used only the patient's first isolate. We excluded cases that required admission to the hospital, febrile UTIs, patients using indwelling urinary catheter or intermittent catheterization, known patients with urachus, vesicoureteral reflux grades 3 to 5, hydronephrosis, history of antibiotic use in the previous 7 days or use of prophylactic antibiotic, visits with a history of urinary stones, urinary neoplasm, patients with asymptomatic bacteriuria, patients with low colony count in urine culture, patients with 3 or more documented episodes of UTI within 6 months, patients that had their antibiotics stopped 48 hours after urine cultures were finalized, patients with coexisting infection-such as streptococcal pharyngitis, otitis media, sinusitis, community-acquired pneumonia, cellulitis, or skin abscess-patients with kidney transplant, patients on induction chemotherapy, neutropenic patients with absolute counts less than $\left(<1.5 \times 10^{9} / L\right)$, patients on prednisone dose of $20 \mathrm{mg} /$ day or $2 \mathrm{mg} /$ 
$\mathrm{kg}$ /day for more than 14 days, and female patients who were pregnant at the time of ambulatory visit. The flow chart summarizing the included and excluded patients is shown in Figure 1.

Susceptibility Testing. Our hospital microbiology laboratory follows Clinical and Laboratory Standards Institute minimal inhibitory concentrations (MIC) breakpoints when interpreting antimicrobial susceptibility. The MICs were determined by the VITEK 2 system (BioMérieux, St Louis, MO) for a maximum of 12 antibiotics selected by microbiologists, pharmacists, and infectious disease specialists. Antimicrobial susceptibility analysis was not reported on duplicate or polymicrobial isolates growing in the same culture.

Antibiotics. We reviewed empirical antibiotics prescribed, dosages, duration, and de-escalation after the identification of microorganisms, and the recognition of antibiotic susceptibility within 48 hours of the initial visit. Telephone encounters and revisits were reviewed within 7 days of treatment to document any treatment used to interpret susceptibility to failure. We reported use of cephalexin, amoxicillin, amoxicillin-clavulanate, trimethoprim-sulfamethoxazole (TMP-SMX), nitrofurantoin, and fluoroquinolones. Second- and third-generation cephalosporins, amoxicillin-clavulanate, and quinolones were considered broad-spectrum antibiotics. Amoxicillin, cephalexin, TMP-SMX, and nitrofurantoin were considered first-line antibiotics. ${ }^{20} \mathrm{~A}$ regimen was considered appropriate if it used first-line antibiotic for the susceptible isolate and was dosed as recommended by the American Academy of Pediatrics. ${ }^{22,23}$ The duration of therapy was determined for all included patients. The accepted duration of treatment was 3 to 5 days, and long antibiotic duration was defined as 7 or more days. The patients' drug allergies, contraindications, and renal function were factored in to assess appropriateness of antibiotic selection.

Statistical Analysis. In descriptive analyses, we reported percentages and frequencies for categoric variables, and mean and SD for continuous variables. Chi-square tests were used to examine the provider distribution, and the proportion of long antibiotic treatment duration among patients with appropriate and inappropriate UTI diagnosis.

Cost Estimation. The price of antibiotics was based on the US average whole sale price data in 2016. This is used for benchmarking only. The total number of milliliters dispensed for treatment was estimated by the product of the antibiotic daily dose and the days of treatment. Generic prices of antibiotics were used as a reference to estimate the cost of antibiotic prescriptions for patients who received an inappropriate diagnosis in this study.

\section{Results}

A total of 178 patients who received a diagnosis of uncomplicated UTI and were treated with antibiotics
Table 1. Characteristics of All Included Outpatients ( $N=178$ ) in Children Ages 2-18 Years

Characteristics

Result

Age, yr, mean \pm SD

$10.3 \pm 4.8$

Female sex, $\mathrm{n}(\%)$

$167(93.8)$

Race, $\mathrm{n}(\%)$

White

167 (93.8)

African American

6 (3.4)

Hispanic

Other

Primary care physician, $\mathrm{n}(\%)$

Pediatrician

Family medicine

Emergency doctor

Nurse practitioner

Physician assistant

Visit site, $\mathrm{n}(\%)$

Emergency

Prompt 103 (57.9)

Clinic

Insurance type, n (\%)

Medicaid

Private

Pyuria, n (\%)*

Negative

18 (10.2)

Positive

$158(89.8)$

*2 missing values.

were included in the study for the determined period. Characteristics of the included patients and their visits are shown in Table 1. Around $94 \%$ of uncomplicated UTI cases treated with antibiotics occurred in white females ( $n=167$ ). Most of the patients had private medical insurance (70.2\%).

Of all patients, 70\% received an inappropriate diagnosis and were treated with antibiotics $(n=125)$. Urine cultures showed polymicrobial growth $(58 \%, n$ $=72$ ), showed skin flora growth $(10.4 \%, n=13)$, or had no growth (32\%, $n=40)$, in cases diagnosed inappropriately $(n=125)$. Antibiotics prescribed mostly in this group were TMP-SMX $(53 \%, n=66)$, cephalexin $(22 \%, n$ $=27)$, and cefdinir $(6 \%, n=7)$. All antibiotics prescribed were not discontinued after urine culture results were finalized in this group. Only $30 \%$ of all included patients were appropriately diagnosed $(n=53)$. Escherichia coli was isolated in $83 \%$ of urine cultures in this group ( $n=$ 44 ), with reported sensitivity of $100 \%$ to nitrofurantoin, $96 \%$ to cefazolin, and $83 \%$ to TMP-SMX. Of all appropriate diagnosis patients ( $n=53$ ), $26 \%$ were treated inappropriately with wide-spectrum antibiotics $(n=8)$, 
Figure 2. Flowchart showing antibiotic prescription for all cases with a diagnosis of uncomplicated urinary tract infection $(\mathrm{N}=178)$.

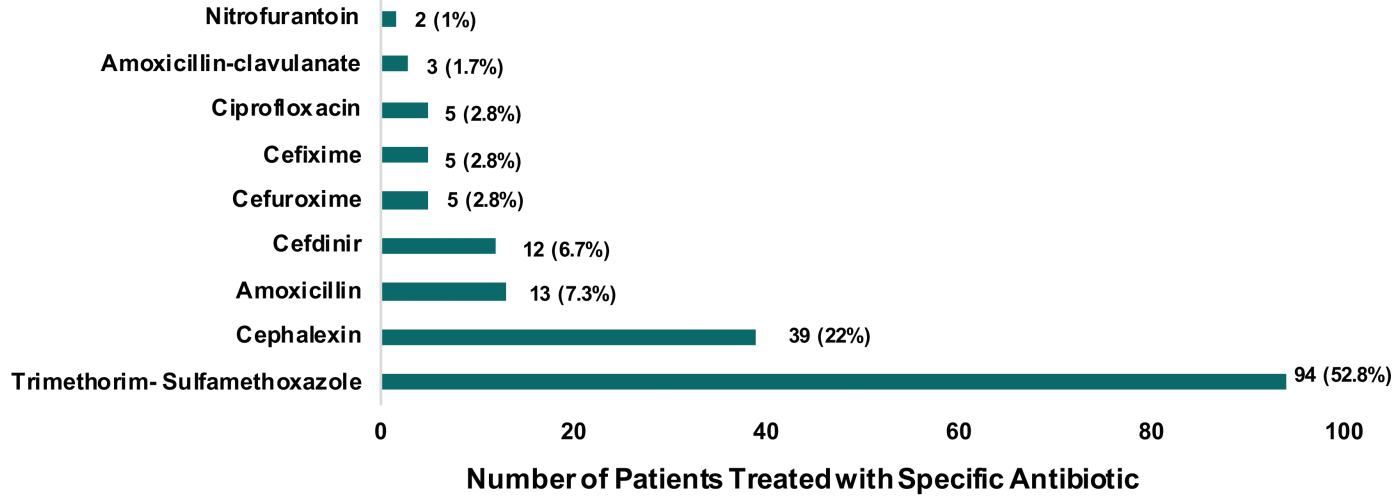

or with low calculated dose $(n=6)$. TMP-SMX $(53 \%, n=$ 28 ) and cephalexin (23\%, $n=12)$ were prescribed mostly for patients with appropriate diagnosis and treatment for uncomplicated UTI.

Cefdinir was prescribed most frequently in patients with an appropriate diagnosis but treated with widespectrum antibiotics for uncomplicated UTI $63 \%, n$ $=5)$. No de-escalation of antibiotics was done after urine culture results were released and showed susceptibility of the isolates to first-line antibiotics. Figure 2 shows that wide-spectrum antibiotics (ciprofloxacin, cefuroxime, cefixime, cefdinir, amoxicillin clavulanate) were prescribed empirically in $16.8 \%(n=30)$ of all cases included in this study awaiting urine culture results, with cefdinir being used most commonly $(n=12)$. Antibiotics were prescribed for 7 or more days in most cases whether they were appropriately or inappropriately diagnosed as demonstrated in Table 2, and there was no statistical significance among both groups $(86.8 \%$ and $77 \%$; $p=0.129$ ).

The estimated cost of antibiotic treatment for the inappropriate diagnosis group ( $n=125$ ) was $\$ 10,755.87$. Most cases of uncomplicated UTI were diagnosed in prompt care and outpatient clinics (57.9\%, 41.6\% consecutively). Uncomplicated UTI was diagnosed inappropriately in $75.5 \%$ of cases evaluated in prompt care $(\mathrm{n}=$ 103), and in $62.2 \%$ of cases seen in outpatient clinic (n =74), with no statistical significance between ambulatory sites $(p=0.052)$. Furthermore, Table 3 shows that there was no statistical difference among the providers' specialty in diagnosing UTI in both groups $(p=0.696)$, whether it was done appropriately or inappropriately.

\section{Discussion}

Uncomplicated UTI occurs usually in children and adolescents with no underlying anatomic or physiologic abnormalities. Lower urinary tract symptoms with evidence of inflammation are essential in establishing the diagnosis of UTI, but quantitative urine culture is the standard method for confirming the infection. ${ }^{20}$ In our study, uncomplicated UTI was diagnosed inappropriately in $70 \%$ of cases based on the results of urine cultures. Urine cultures grew polymicrobial species or skin flora contaminants in most of the collected samples, which reflects nonsterile methods of urine collection. This led to unnecessary antibiotic treatment, and hence extra cost. Additionally, antibiotics were not discontinued after urine culture results were finalized, even in the group that had no growth $(32 \%, n=40)$. This might be explained by the lack of follow-up on urine culture results or misconceptions about the utility of urine culture results in confirming the diagnosis of UTI.

Only $30 \%$ of all included patients were appropriately diagnosed $(n=53)$. The bacteria isolated most commonly in this group was $E$ coli, with a frequency of $83 \%$. Our isolates were highly sensitive to nitrofurantoin (100\%) and cefazolin (96\%), and, to a lesser extent, TMP-SMX (83\%). It seems that TMP-SMX and cephalexin were largely selected empirically by providers to treat presumed uncomplicated UTIs in our study. Although a study of national uropathogen resistance rates in pediatric UTIs

Table 2. Days of Antibiotics Prescribed for All Included Patients $\left(x^{2}=2.30, p=0.129\right)$

\begin{tabular}{lc} 
Days of Antibiotics & $\begin{array}{c}\text { Appropriately Diagnosed } \\
(\mathbf{n}=\mathbf{5 3}), \mathbf{n o} \text {. }(\%)\end{array}$ \\
$<7$ days & $46(86.7)$ \\
$\geq 7$ days & $7(13.3)$ \\
\hline
\end{tabular}




\begin{tabular}{|c|c|c|}
\hline Provider & $\begin{array}{l}\text { Appropriately Diagnosed } \\
\text { (n= 53), no. (\%) }\end{array}$ & $\begin{array}{l}\text { Inappropriately Diagnosed } \\
\qquad(\mathrm{n}=125), \text { no. (\%) }\end{array}$ \\
\hline Pediatrician & $17(32.1)$ & $29(23.2)$ \\
\hline Family medicine & $14(26.4)$ & $34(27.2)$ \\
\hline Nurse practitioner & $11(20.8)$ & $32(25.6)$ \\
\hline Physician assistant & $8(15.1)$ & $25(20)$ \\
\hline Emergency medicine & $3(5.7)$ & $5(4)$ \\
\hline
\end{tabular}

found that more than $96 \%$ of $E$ coli isolates in female patients were sensitive to first-generation cephalosporin, TMP-SMX was still prescribed most frequently (49\% of visits) in ambulatory pediatric UTIs. ${ }^{4}$ Our study showed similar results, in which TMP-SMX was prescribed empirically in $52.8 \%(n=94)$ of all included cases $(n=178)$, and cephalexin was prescribed less commonly (21.9\%), whereas nitrofurantoin was prescribed only in $1.0 \%$ of all patients $(n=2)$. Only $16.8 \%(n=30)$ of all included patients in our study were treated empirically with wide-spectrum antibiotics; however, cefdinir was still frequently prescribed $(n=12)$. The reason why providers tend to prescribe cefdinir more commonly might be related to studies reporting high resistance of uropathogens to first-line antibiotics used in the pediatric population in the United States and globally. ${ }^{24,25}$ The lack of antibiograms designed specifically for Gram-negative isolates in the urine in our pediatric ambulatory settings contributes to selection of wide-spectrum antibiotics. In addition, antibiograms derived from hospital-based laboratory data or adult ambulatory data will not provide accurate information about antibiotic resistance patterns for pediatric patients in the ambulatory settings. ${ }^{26}$ Nitrofurantoin and cephalexin could have been used more empirically in our population if antibiograms were designed specifically for our pediatric ambulatory sites.

Of all included patients, $80 \%(n=142)$ in our study were treated with antibiotics for more than 7 days, whether they received an appropriate or inappropriate diagnosis. This might be related to providers following recommendations of longer duration of treatment for complicated febrile UTIs. The American Academy of Pediatrics published guidelines for diagnosis and treatment of initial febrile UTIs in children 2 months to 2 years and recommended 7 to 14 days of treatment. ${ }^{20}$ However, no specific guidelines were developed for uncomplicated cystitis in children of older age groups. Uncomplicated UTIs in children can be treated adequately with shorter durations of antibiotics. A Cochrane review found that short courses of oral antibiotics (2-4 days) are as effective as standard courses (7-14 days) at sterilizing urine cultures without increases in recurrence or the development of resistance at the end of therapy. ${ }^{27}$ Inappropriate diagnosis and treatment of uncomplicated UTIs leads to unnecessary cost. We estimated that $\$ 10,755.87$ was spent on antibiotic prescription only in this group, whether first-line or wide-spectrum antibiotics were used.

Our study had some limitations. First, it was conducted retrospectively covering a short period of time. Although we reviewed cases in the peak of winter season, when illnesses were common and the usual demand on antibiotics might be high, patients who had associated infections were excluded from our study, which makes our results very reliable. Secondly, we could not identify from the electronic records whether urine samples were collected after providers assessed their patients, at the time when the patients were triaged, or through midstream clean catch specimens. Random specimens tend to give falsely positive or negative results depending on the dilution of the urine and the cleanness of the method of collection. Thirdly, we could not calculate cost accurately because health insurance plans differed among patients, copayments versus actual insurance coverage policies were unknown, and different outpatient pharmacies were used.

\section{Conclusions}

There is a lack of appropriate diagnosis and treatment of uncomplicated UTI in pediatric patients in our institution. We need to develop antibiograms designed for pediatric outpatient isolates, specifically for uncomplicated UTIs to improve empirical antibiotic choices and limit unnecessary use of third-generation cephalosporins. Nitrofurantoin, cephalexin, and TMP-SMX can be used empirically for uncomplicated acute cystitis in our outpatient pediatric population with no risk factors. Future prospective studies are needed to assess the efficacy of short antibiotic regimens for uncomplicated UTIs. Education of providers about the appropriate diagnosis and treatment of uncomplicated UTI in children is essential in our pediatric ambulatory care settings. The ASPs should target pediatric outpatient sites in our institution to promote judicious antibiotic use, to help in reducing selection of bacterial resistance, and to minimize the cost of therapy.

\section{ARTICLE INFORMATION}

Affiliations Pediatric Infectious Diseases (BAS), Department of Pediatrics, University of Illinois College of Medicine at Peoria and Children's Hospital of Illinois, Peoria, Illinois, Department 
of Surgery (JL), Huntington Hospital, Pasadena, California, University of Illinois College of Medicine at Peoria (MMA, BB), Peoria, Illinois, Center for Outcomes Research (JR), University of Illinois College of Medicine at Peoria, Peoria, Illinois, Department of Health Sciences Education (RT), University of Illinois College of Medicine at Peoria, Peoria, Illinois, Academic Affairs (MA), Internal Medicine, University of Illinois College of Medicine at Peoria, Peoria, Illinois

Correspondence Ban AL-Sayyed, MD; banhusni@uic.edu

Accepted August 11, 2018

Disclosure The authors declare no conflicts or financial interest in any product or service mentioned in the manuscript, including grants, equipment, medications, employment, gifts, and honoraria. The authors had full access to all the data and take responsibility for the integrity and accuracy of the data analysis.

Copyright Published by the Pediatric Pharmacy Advocacy Group. All rights reserved.

For permissions, email: matthew.helms@ppag.org

\section{REFERENCES}

1. Freedman AL. Urologic diseases in North America Project: trends in resource utilization for urinary tract infections in children. J Urol. 2005;173(3):949-954.

2. Abbo LM, Hooton TM. Antimicrobial stewardship and urinary tract infections. Antibiotics. 2014;3(2):174-192.

3. Watson JR, Sánchez PJ, Spencer JD, et al. Urinary tract infection and antimicrobial stewardship in the emergency department. Pediatr Emerg Care. 2018;34(2):93-95.

4. Copp HL, Shapiro DJ, Hersh AL. National ambulatory antibiotic prescribing patterns for pediatric urinary tract infection, 1998-2007. Pediatrics. 2011;127(6):1027-1033.

5. Fleming-Dutra, K, Hersh AL, Shapiro DJ, et al. Prevalence of inappropriate antibiotic prescriptions among US ambulatory care visits, 2010-2011. JAMA. 2016;315(17):18641873.

6. Centers for Disease Control and Prevention (CDC). Office-related antibiotic prescribing for persons aged $\leq 14$ years--United States, 1993-1994 to 2007--2008. MMWR. 2011;60(34):1153-1156.

7. Pichichero ME. Dynamics of antibiotic prescribing for children. JAMA. 2002;287(23):3133-3135.

8. Shapiro DJ, Hicks LA, Pavia AT, et al. Antibiotic prescribing for adults in ambulatory care in the USA, 2007-09. J Antimicrob Chemother.2014;69(1):234-240.

9. Suda KJ, Hicks LA, Roberts RM, Hunkler RJ, Danziger $\mathrm{LH}$. A national evaluation of antibiotic expenditures by healthcare setting in the United States, 2009. J Antimicrob Chemother. 2013;68(3):715-718.

10. Rosenberg DJ. Infections, bacterial resistance, and antimicrobial stewardship: the emerging role of hospitalists. J Hosp Med. 2012;7(suppl 1):S34-S43.

11. Camins BC, King MD, Wells JB, et al. Impact of an antimicrobial utilization program on antimicrobial use at a large teaching hospital: a randomized controlled trial. Infect Control Hosp Epidemiol. 2009;30(10):931-938.
12. Ingram PR, Seet JM, Budgeon CA, Murray R. Point-prevalence study of inappropriate antibiotic use at a tertiary Australian hospital. Intern Med J. 2012;42(6):719-721.

13. Levin PD, Idrees $S$, Sprung $C L$, et al. Antimicrobial use in the ICU: indications and accuracy-an observational trial. J Hosp Med. 2012;7(9):672-678.

14. Patel SJ, Oshodi A, Prasad P, et al. Antibiotic use in neonatal intensive care units and adherence with Centers for Disease Control and Prevention 12 step campaign to prevent antimicrobial resistance. Pediatr Infect Dis J. 2009;28(12):1047-1051.

15. Dellit TH, Owens RC, McGowan JE Jr, et al. Infectious Diseases Society of America and the Society for Healthcare Epidemiology of America guidelines for developing an institutional program to enhance antimicrobial stewardship. Clin Infect Dis. 2007;44(2):159-177.

16. Fridkin SK, Baggs J, Fagan R, et al. Vital signs: improving antibiotic use among hospitalized patients. MMWR. 2014;63(09):194-200.

17. Hersh AL, Shapiro DJ, Pavia AT, et al. Antibiotic prescribing in ambulatory pediatrics in the United States. Pediatrics. 2011;128(6):1053-1061.

18. Vaz LE, Kleinman KP, Raebel MA, et al. Recent trends in outpatient antibiotic use in children. Pediatrics. 2014;133(3):375-385.

19. Hooton TM, Stamm WE. Diagnosis and treatment of uncomplicated urinary tract infection. Infect Dis Clin North Am. 1997;11(3):551-581.

20. Subcommittee on Urinary Tract Infection, Steering Committee on Quality Improvement and Management; Roberts KB. Urinary tract infection: clinical practice guideline for the diagnosis and management of the initial UTI in febrile infants and children 2 to 24 months. Pediatrics 2011;128(3):595-610.

21. Hoberman A, Wald ER, Penchansky L, Reynolds EA, Young S. Enhanced urinalysis as a screening test for urinary tract infection. Pediatrics. 1993;91(6):1196-1199.

22. American Academy of Pediatrics. Antibacterial drugs for pediatric patients beyond the newborn period. In: Kimberlin DW, Brady MT, Jackson MA, Long SS, eds. 2015 Report of the Committee on Infectious Diseases. 30th ed. Elk Grove Village, IL: American Academy of Pediatrics; 2015:884.

23. Wald ER. Cystitis and pyelonephritis. In: Cherry JD, Harrison GJ, Kaplan SL, et al, eds. Feigin and Cherry's Textbook of Pediatric Infectious Diseases. 7th ed. Philadelphia, PA: Elsevier Saunders; 2014:535.

24. Gaspari RJ, Dickson E, Karlowsky J, et al. Antibiotic resistance trends in paediatric uropathogens. Int J Antimicrob Agents. 2005;26(4):267-271.

25. Bryce A. Global prevalence of antibiotic resistance in paediatric urinary tract infections caused by Escherichia coli and association with routine use of antibiotics in primary care: systematic review and meta-analysis. BMJ. 2016;352-363.

26. Dahle KW, Korgenski EK, Hersh AL, et al. Clinical value of an ambulatory-based antibiogram for uropathogens in children. J Pediatric Infect Dis Soc. 2012;1(4):333-336.

27. Fitzgerald A, Mori R, Lakhanpaul M, Tullus K. Antibiotics for treating lower urinary tract infection in children. Cochrane Database Syst Rev. 2012;15(8):CD006857. 